\title{
Contrast-enhanced echocardiography to rule-out active intrapericardial bleeding following coronary artery perforation
}

\author{
Francesco Moroni ${ }^{1}$, Valeria Magni ${ }^{1}$, Alberto Cappelletti ${ }^{1}$, Cristina Capogrosso ${ }^{2}$, \\ Cosmo Godino $^{1}$, Matteo Montorfano ${ }^{3 *}$, Lorenzo Azzalini ${ }^{3 *}$ \\ ${ }^{1}$ Cardiology Division, Cardio-Thoracic-Vascular Department, San Raffaele Scientific Institute, Milan, Italy \\ ${ }^{2}$ Echocardiography Division and Coronary Care Unit, Cardio-Thoracic-Vascular Department, \\ San Raffaele Scientific Institute, Milan, Italy \\ ${ }^{3}$ Interventional Cardiology Division, Cardio-Thoracic-Vascular Department, \\ San Raffaele Scientific Institute, Milan, Italy
}

A 66-year-old man underwent percutaneous coronary intervention on a stenosis of the ramus intermedius for worsening dyspnea and positive exercise testing (Fig. 1A). Weight-adjusted heparin (a total of $8000 \mathrm{IU}$ ) was administered as per standard practice. Two drug-eluting stents were implanted at the ostial and proximal segments of the vessel. Final angiography demonstrated a flow-limiting dissection, which was covered with a stent. Upon subsequent contrast injection, an Ellis type 3 coronary artery perforation (CAP) was observed (Fig. 1B). A balloon was inflated proximal to the CAP. Severe hypotension arose, and transthoracic echocardiogram (TTE) showed cardiac tamponade. Pericardiocentesis was performed, with complete resolution of the effusion. A right femoral artery access was secured, and a second guide catheter engaged the left coronary artery (ping-pong technique) (Fig. 1C). Three 3-mm MicroNester 18 (Cook Medical, Bloomington, Indiana) coils were delivered to seal the perforation through the second guide catheter (Fig. 1D, E). The patient was transferred to the intensive care unit. No anticoagulation reversal with protamine was deemed necessary since CAP sealing with coils was considered adequate. Two hours later, de novo formation of pericardial effusion was documented, and additional $150 \mathrm{~mL}$ of blood were extracted from the pericardial drainage. Suspecting active intrapericardial bleeding, a new coronary angiogram was performed, which did not identify bleeding from the CAP (Fig. 1F). Contrastenhanced TTE was performed after intravenous injection of $10 \mathrm{~mL}$ of sodium hexafluoride-based ultrasound contrast (SonoVue, Bracco Imaging, San Donato Milanese, Italy). Echocardiographic contrast is strictly intravascular, and is visualized as hyperechoic spots using dedicated imaging protocols. The identification of contrast in the pericardial space implies active extravasation. No evidence of contrast in the pericardial space was detected in our patient, ruling out active bleeding (Fig. 1G, H). The patient remained subsequently stable until hospital discharge. The Supplementary Movie 1 presents the key passages of this case, including CAP management and contrast-enhanced echocardiography.

Conflict of interest: Lorenzo Azzalini - honoraria from Abbott Vascular, Guerbet, Terumo, and Sahajanand Medical Technologies; and research support from ACIST Medical Systems, Guerbet, and Terumo. None of the funding source had any role or contributed in any way to the present work.

Address for correspondence: Lorenzo Azzalini, MD, PhD, MSc, Interventional Cardiology Division, Cardio-Thoracic-Vascular Department, San Raffaele Scientific Institute, Via Olgettina 60, 20132 Milan, Italy, tel: +39 0226437331 , fax: +39 0226437339, e-mail: azzalini.lorenzo@hsr.it

Received: 5.06.2019 Accepted: 5.11.2019

*These Authors share last authorship. 

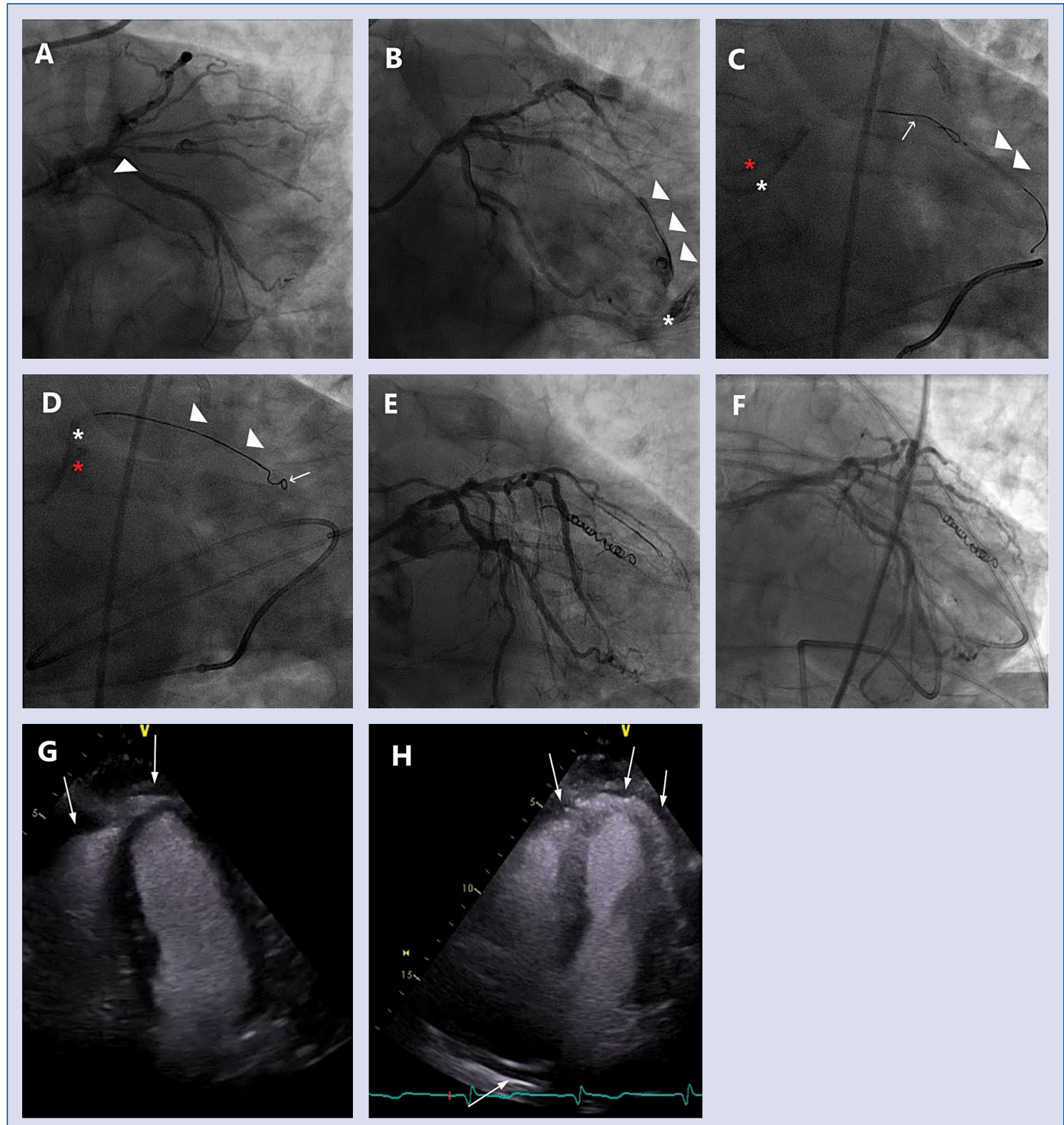

Figure 1. Contrast-enhanced echocardiography to rule-out active intrapericardial bleeding following coronary artery perforation. A. Critical stenosis of the ramus intermedius. The arrowhead points at the site of the stenosis; B. Ellis type 3 coronary artery perforation (CAP). The arrowheads point at the site of the perforation, while the asterisk marks contrast extravasation into the pericardial space; C. Ping-pong technique to achieve balloon occlusion and subsequent CAP embolization. The arrowhead points at the balloon inflated proximally to the CAP through the first guiding catheter. The arrow points at the microcatheter and guidewire through the second guiding catheter. Red asterisk: guiding catheter via the left radial access; white asterisk: guiding catheter via the right femoral access; D. Coil deployment in the ramus intermedius. Arrowheads: microcatheter. Arrow: coil; E. Complete deployment of the coils and complete sealing of the CAP; F. Second-look coronary angiography, showing no active bleeding from the CAP; G. Transthoracic contrast-enhanced echocardiogram (4-chamber view), showing no contrast in the pericardial space (arrows); H. Transthoracic contrast-enhanced echocardiogram (right ventricle-focused 4-chamber view), showing no contrast in the pericardial space (arrows). 